\title{
Polymer chain scission at constant tension - an example of force-induced collective behaviour
}

\author{
Jaroslaw Paturej ${ }^{1,2}$ (a), Andrey Milchev ${ }^{1,3}$, Vakhtang G. Rostiashvili $^{1}$ and Thomas A. Vilgis ${ }^{1}$ \\ 1 Max Planck Institute for Polymer Research, 10 Ackermannweg, 55128 Mainz, Germany \\ Institute of Physics, University of Szczecin, Wielkopolska 15, 70451 Szczecin, Poland \\ Institute for Physical Chemistry, Bulgarian Academy of Sciences, 1113 Sofia, Bulgaria
}

PACS 82.37.-j - Single molecules kinetics

PACS 82.35.Lr - Physical properties of polymers

PACS 05.40.-a - Fluctuation phenomena, random processes, and Brownian motion

\begin{abstract}
The breakage of a polymer chain of segments, coupled by anharmonic bonds with applied constant external tensile force is studied by means of Molecular Dynamics simulation. We show that the mean life time of the chain becomes progressively independent of the number of bonds as the pulling force grows. The latter affects also the rupture rates of individual bonds along the polymer backbone manifesting the essential role of inertial effects in the fragmentation process. The role of local defects, temperature and friction in the scission kinetics is also examined.
\end{abstract}

Introduction. - The understanding of a great variety of phenomena related to stability, fracture, and elastic behavior of materials requires fundamental knowledge of the intermolecular dynamics of bond breakage. In most cases scission of bonds may be caused by mechanical load, irradiation, or just increase in temperature. Examples related to this field are diverse and include mechanical fracture of materials [1,2, polymer rupture [3 4, adhesion [8, friction 9, mechanochemistry [10,11. Recently, there has been an enormous increase of interest in polymer fragmentation due to the possibility of biomolecule's micromanip- ulation in experiments using force spectroscopy methods 12 14. This has motivated also theoretical investigations and computer experiments [15].

In particular, the problem of polymer fragmentation has got a longstanding history in scientific literature. The treatment of bond rupture as a kinetic process dates back to the publications of Bueche [16] and Zhurkov et al. [17]. In the recent years these seminal papers have been complemented by a variety of computer experiments. Molecular Dynamics (MD) simulations of chain rupture at constant stretching strain has been carried out, whereby harmonic 18, 19, Morse [20, 22, or Lennard-Jones [23, 26] interactions have been employed. A theoretical interpretation of $\mathrm{MD}$ results, based on an effectively one-particle model (Kramers rate theory) has been suggested [25,26]. On the

(a) E-mail: jpaturej@univ.szczecin.pl other hand, an analytical treatment of a polymer fragmentation under constant stress have been proposed in terms of many-particle version of transition state theory [22].

Recently, we proposed a description of linear polymer scission under constant tensile force by using the multidimensional Langer-Kramers theory, which was found to compare favorably with the results of MD simulations [27]. Within this approach a single bond rupture is seen as a thermally activated escape from the bottom of a potential well. The life time $\tau$ before a bond scission takes place, is determined by diffusive crossing of an energy barrier $E_{b}$ that is reduced under the applied external force $f$. The adopted theoretical treatment assumes a single collective unstable modes as being mainly responsible for chain breakage. Such unstable mode peaks around an "endangered" bond of negative spring constant and decays exponentially towards both chains end. Similar collectivity effect has also been reported in the case of ring polymers stretched with constant strain [28].

In this letter we report some new results pertaining to the rupture kinetics of single $1 D$ and $3 D$ polymer chains induced by constant tensile stress in a broad interval of pulling forces.

The model. - As in our previous work [27, we use a coarse-grained model of a polymer chain of $N$ beads connected by bonds, whereby each bond of length $b$ is described by a Morse potential $V^{\mathrm{M}}(r)=D\{1-\exp [-a(r-$ 
b)] $\}^{2}$, with $a$ being a constant, $a=1$, that determines bond elasticity,

The dissociation energy $D$ of a given bond is measured in units of $k_{B} T$, where $k_{B}$ is the Boltzmann constant and $T$ denotes temperature. Since $V^{\mathrm{M}}(0) \approx 2$, the Morse potential is only weakly repulsive and segments could partially penetrate one another at $r<b$. Therefore, in order to allow properly for the excluded volume interactions between bonded particles, we take the bond potential as a sum of $V^{\mathrm{M}}$ and the so called Weeks-Chandler-Anderson (WCA) potential $V^{\mathrm{WCA}}=4 \epsilon\left[\left(\frac{\sigma}{r}\right)^{12}-\left(\frac{\sigma}{r}\right)^{6}+\frac{1}{4}\right] \theta\left(2^{1 / 6} \sigma-\right.$ $r)$, with $\theta(x)=0$ or 1 for $x<0$ or $x \geq 0$, and $\epsilon=1, \sigma=1$. The parameter $\sigma$ sets up the length scale of equilibrium monomer size $b=2^{1 / 6} \sigma \approx 1.12$. The nonbonded interactions between monomers are also taken into account by means of the WCA potential.

The dynamics of the chain is obtain by solving a Langevin equation for the position $\mathbf{q}_{n}=\left[x_{n}, y_{n}, z_{n}\right]$ of each bead in the chain, $m \ddot{\mathbf{q}}_{n}=\mathbf{F}_{n}^{\mathrm{M}}+\mathbf{F}_{n}^{\mathrm{WCA}}-\gamma \dot{\mathbf{q}}_{n}+\mathbf{R}_{n}(t)+$ $\mathbf{f}_{n} \delta_{n N}(n, \ldots, N)$, which describes the Brownian motion of a set of bonded particles whereby the last of them is subjected to external stretching force $\mathbf{f}=[f, 0,0]$. The influence of solvent is split into slowly evolving viscous force and rapidly fluctuating stochastic force. The random, Gaussian force $\mathbf{R}_{n}$ is related to friction coefficient $\gamma$ by the fluctuation-dissipation theorem. It should be noted that we consider only grafted chains, i.e. one of the chain ends is fixed in space. The integration step is 0.002 time units (t.u.) and time in measured in units of $\sqrt{m / \sigma^{2} D}$, where $m$ denotes the mass of the beads, $m=1$.

We start the simulation with all beads placed at distance $b$ from each other, and then we let the chain to equilibrate in the Langevin heat bath. Due to the presence of the external pulling force, the equilibrium configuration of the chain is more or less stretched and deviates markedly from coil shape. Once equilibration is achieved, time is set to zero and one measures the elapsed time $\tau$ before any of the bonds exceeds certain extension $r_{h}$, which sets the criterion for considering such bond broken. We use a large value for the critical bond extension, $r_{h}=5 b$, which is defined as a threshold to a broken state. This convention is based on our checks that the probability for recombination of bonds, stretched beyond $r_{h}$, is vanishingly small. We repeat this procedure for a large number of events $5 \times 10^{4}$ so as to determine the mean rupture time $\langle\tau\rangle$ which we refer as Mean First Breakage Time (MFBT). The details of this method can be found in Ref. [27.

MD-results. - In our computer experiments we focused on the following most salient properties of the bond breakage process:

Chain length dependence of the MFBT $\langle\tau\rangle$. In Fig. 11a) and the inset of Fig. 1 a) we present numerical results for $\langle\tau\rangle$ as a function of the number of beads $N$ for chains that are stretched in the interval $0.1 \geq f \geq 0.3$ of pulling forces both in $1 D$ and $3 D$. Regardless of dimensionality of the examined systems, for a given value of $f$ one ob-
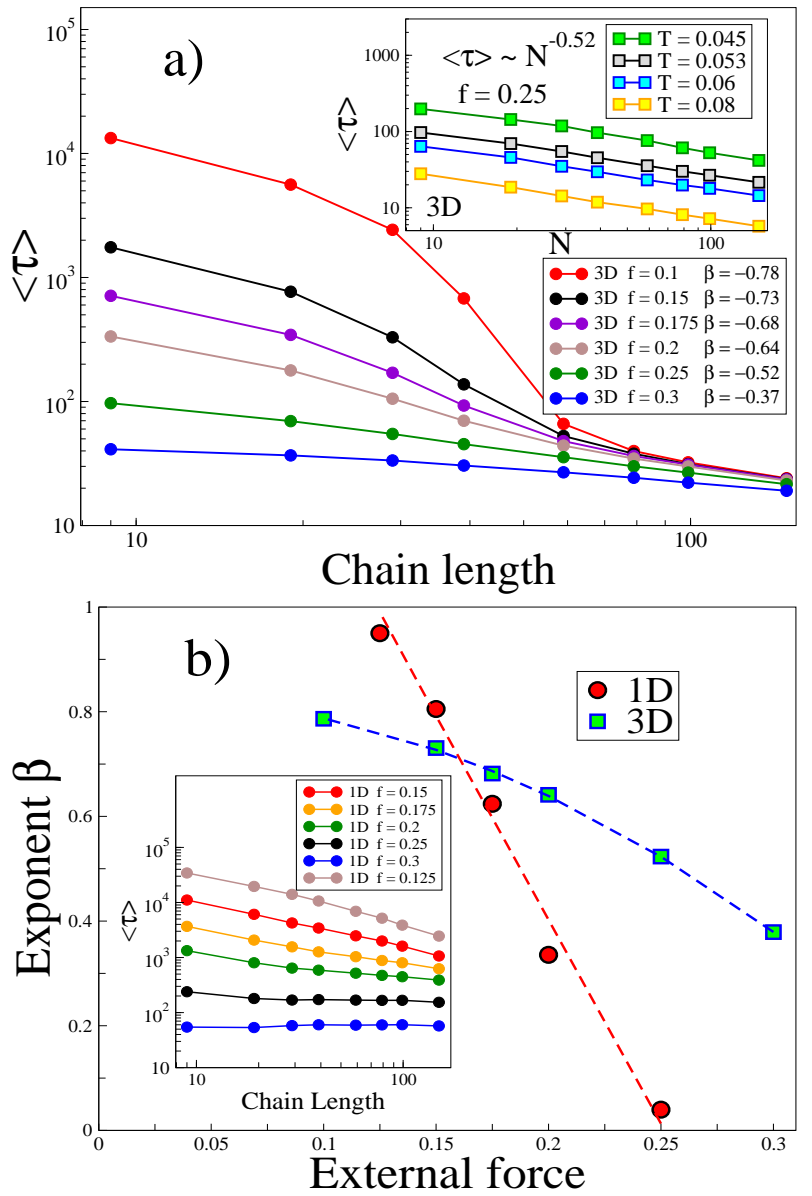

Fig. 1: a) Mean first breakage time $\langle\tau\rangle$ vs. $\quad N$ for a $3 D$ chain. In the legend slopes of fitting lines $\langle\tau\rangle \propto N^{-\beta}$ are presented which were found in the range $N=80-150$. The inset shows $\langle\tau\rangle$ against $N$ for $3 D$ chains stretched by force $f=0.25$ at different temperatures. b) Variation of slope $\beta$ with external pulling force $f$ for chains in $1 D$ and $3 D$. The inset shows $\langle\tau\rangle$ vs. $N$ for a $1 D$ chain. Parameters of the heat bath are temperature $T=0.53$ and friction $\gamma=0.25$.

serves a power-law decrease, $\langle\tau\rangle \propto N^{-\beta}$. This relationship is found for sufficiently long chains (asymptotic limit) $N \gtrsim 80$, where finite-size effects do not play a role. Additionally, from the inset of Fig. 19) we observe that there is no impact of the temperature on the value of the slope $\beta$. Furthermore, Fig. 10) indicates that with growing tensile strength the life time $\langle\tau\rangle$ becomes nearly independent of $N$ which is among the most important results of this study. This independence is fully consistent with our recent findings 27 for relatively strong pulling force, $f=0.25$. It was shown in 27 that in this case the process of bond scission is governed by a collective unstable mode peaked around an "endangered" bond (i.e. a bond with negative spring constant) and decays exponentially towards both chain's ends. On the other hand, in the opposite limit of thermal degradation of polymers $(f=0)$ (i.e., in the socalled thermolysis) the total probability for scission of a polymer with $N$ bonds within a certain time interval is $N$ 
times larger than that for a single bond which is what one would expect if bonds do break entirely at random and independent of one another. The latter leads to the relationship $\langle\tau\rangle \propto N^{-1}$ which has been seen recently in computer simulations of harmonic 29] and anharmonic 30. polymer chain models. Moreover, Fig. 1b) clearly shows that with increasing pulling force $f$ the exponent $\beta$ gradually decreases within the interval $0<\beta<1$. Thus the slope $\beta$ can be treated as a quantitative measure of the degree of cooperativity in rupture events. As the slope $\beta$ decreases, the nature of scission events become more and more collective.

It is pertinent to note that in the literature one finds conflicting data regarding the $\langle\tau\rangle$ vs. $N$ dependence. For example, in [26] it is claimed that $\langle\tau\rangle \propto N^{-1}$, but a more close inspection of the Fig. 4 in this work where the dependence is shown gives for the exponent $\beta=0.17 \div 0.2$. In another work 28, a chain rupture under fixed strain has been investigated by making use the many-body LangerKramers theory and MD-simulation. On the one hand, the authors discuss the presence of collective unstable mode (cf. 27]) but on the other hand they claim that for the total chain again $\langle\tau\rangle \propto N^{-1}$ which should be considered as a hallmark of missing collectivity. Unfortunately, there have been no corresponding simulations which would have confirmed the conclusion made in 28 .

Dependence of MFBT on Pulling Force. The dependence of the MFBT $\langle\tau\rangle$ on external force $f$ for $1 D$ and $3 D$ chains composed of $N=30$ beads is shown in Fig. 2. Evidently for sufficiently strong stretching forces $f \gtrsim 0.175$ an exponential decay $\langle\tau\rangle \propto e^{\left(E_{0}-\alpha f\right) / k_{B} T}$ is observed. The main reason of this is the following: As the pulling force grows, the energy barrier, which separates intact bonds from the broken ones, declines. As a consequence, $\langle\tau\rangle$ decreases. One should note that the parameters $\alpha$ and $E_{0}$ change only slightly with the coupling parameter $\gamma$ of the thermostat. Fig. 2 indicates also a considerable dif-

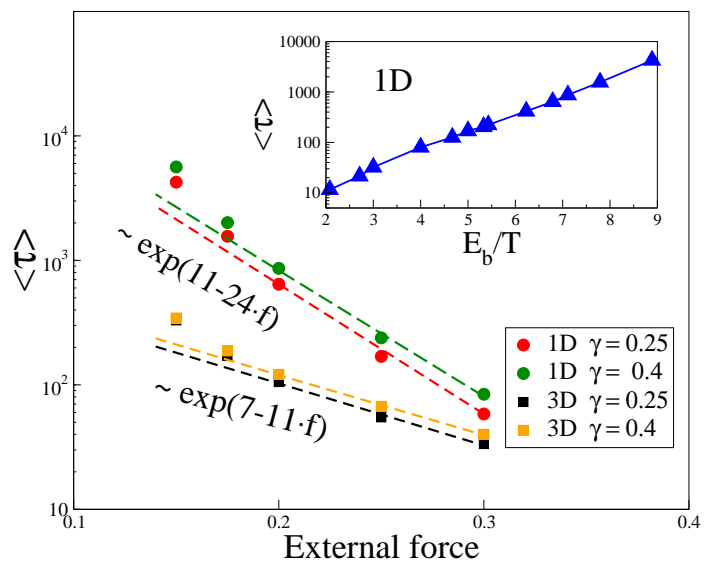

Fig. 2: Force-dependent mean first breakage time for a $1 D$ and $3 D$ chains with $N=30$. The inset shows of $\langle\tau\rangle$ vs. $E_{b} / T$ for $1 D$ system with $T=0.053$ and $\gamma=0.25$.

ference in the values of $\alpha$ between $1 D$ and $3 D$. In the inset of Fig. 2 we present $\langle\tau\rangle$ as a function of the ratio $E_{b} / T$ of the barrier height to temperature. This finding is in agreement with the understanding of the polymer rupture as a thermally activated process [16, 17] and is manifested by an Arrhenian relationship $-\langle\tau\rangle \propto e^{E_{b} / T}$, where $E_{b}=E_{0}-\alpha f$.

Life-time probability distribution $W(t)$. In Fig. 3 we display the probability distribution function $W(t)$ of the observed scission times $t$ for several ratios $E_{b} / T$ of the

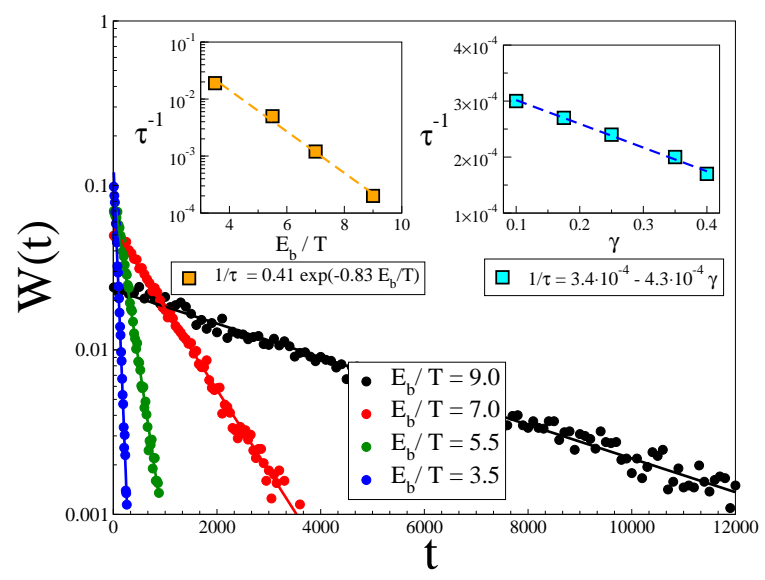

Fig. 3: Life-time probability distributions $W(t)$ for different height of the energy barrier $E_{b} / T$ in $1 D$. Here the chain length is $N=30$, the pulling force $f=0.15$, and $\gamma=0.25$. Symbols denote simulation results and full lines stand for fitting functions $W(t) \propto \exp (-t / \tau)$. The two insets show the dependence of $\tau^{-1}$ on $E_{b} / T$ [left panel], and on $\gamma$ [right panel].

barrier height to temperature in the case of $1 D$ chain composed of $N=30$ beads. It appears that $W(t)$ goes asymptotically as $W(t) \propto e^{-t / \tau\left(E_{b} / T\right)}$ in accordance with our recent findings 27. Additionally from the inset [left panel] of Fig. 3 one may easily verify that the characteristic time goes as $\tau\left(E_{b} / T\right) \propto \exp \left(E_{b} / T\right)$. Moreover, a simple linear relationship has been found for friction dependence of $\tau(\gamma)$ as shown in the inset [right panel] of Fig. 3 .

Rupture probability histograms. In Fig. 4 we show the MFBT $\left\langle\tau_{n}\right\rangle$ of the individual bonds for chains composed of $N=30$ beads in $1 D$ and $3 D$. We compare the results for different pulling forces as indicated in the legend. For the case $f=0.3$ one may readily verify that the bonds located in the vicinity of the grafted bead live nearly twice as long as those close to the loose end where the tensile force is applied, regardless of dimensionality. In contrast, chains stretched by a gentle force, $f=0.15$, display very different distributions of $\left\langle\tau_{n}\right\rangle$. In the case of a $3 D$ system the bonds at the tethered end live on the average significantly $(2 \div 3$ times $)$ shorter than those close to pulled end. Evidently, staring from $n \gtrsim 10$ the lifetime of bonds progressively increases with increasing proximity to the free chain end. In the case of $1 D$ string stretched with $f=0.15$ this effect is missing and $\left\langle\tau_{n}\right\rangle$ is uniformly distributed along the polymer backbone apart from the first 

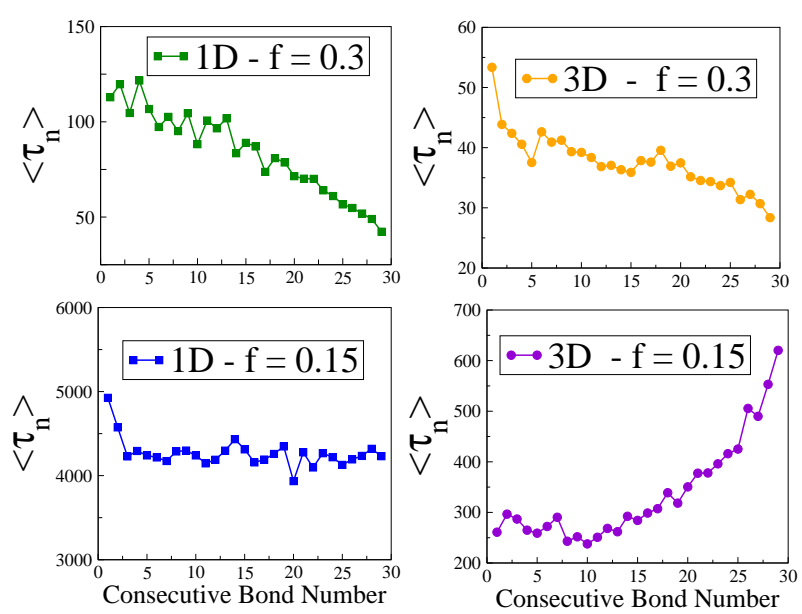

Fig. 4: Variation of the mean life time $\left\langle\tau_{n}\right\rangle$ with consecutive bond number for a 30-particle chain in one and in three dimensions. The polymer is stretched by a force $f=0.15$ or $f=0.3$ at $T=0.053$ and $\gamma=0.25$.

terminal bond which lives $\approx 20 \%$ longer. As expected, all distributions displayed in Fig. 4 are asymmetric due to the constraint imposed on the motion of the first bead in the chain.

In Fig. 5 we present the probability for bond scission of individual bonds in $1 D$ and $3 D$ for several strengths of the pulling force. The histograms display the (normalized) rate at which a certain bond $n$ along the polymer backbone breaks. From the inspection of Fig. 5 a) one sees that the preferential scission of the bonds with particular consecutive bond number essentially depends on the value of force. For strong pulling $f=0.3$ one finds that the terminal bond which is subjected to pulling as well as the bonds in its neighborhood break more frequently than whose around fixed end. Evidently, in this case the rupture rate decreases steadily from the free chain end to the tethered one. A similar scission scenario is visible also for the $1 D$ chain as shown in the inset of Fig. 5 a).

In contrast, as the stretching force is decreased, the corresponding rupture histogram for a $3 D$ chain becomes flatter. For $f=0.2$ the distribution of scission rates becomes uniform exept for the bonds in the vicinity of both ends. A further decrease of the pulling force results in a qualitative change in the distribution. Evidently, for $f=0.15$ the bonds in the middle of the chain, which are also somewhat closer to the fixed chain end, become more vulnerable as compared to those at the chain ends. Note that for the smallest pulling force $(f \lesssim 0.15)$ the rupture histogram already resembles the respective histogram in the case of thermal degradation of a polymer 30] which takes place in the absence of externally induced tension. If two pulling forces are applied simultaneously to both chain ends, Fig. 5b)-d), one finds expectedly a symmetric scission probability distribution regarding bond number, even though $\langle\tau\rangle$ hardly changes - Fig. 5e). Again. the scission rate of the terminal bonds goes down with decreasing
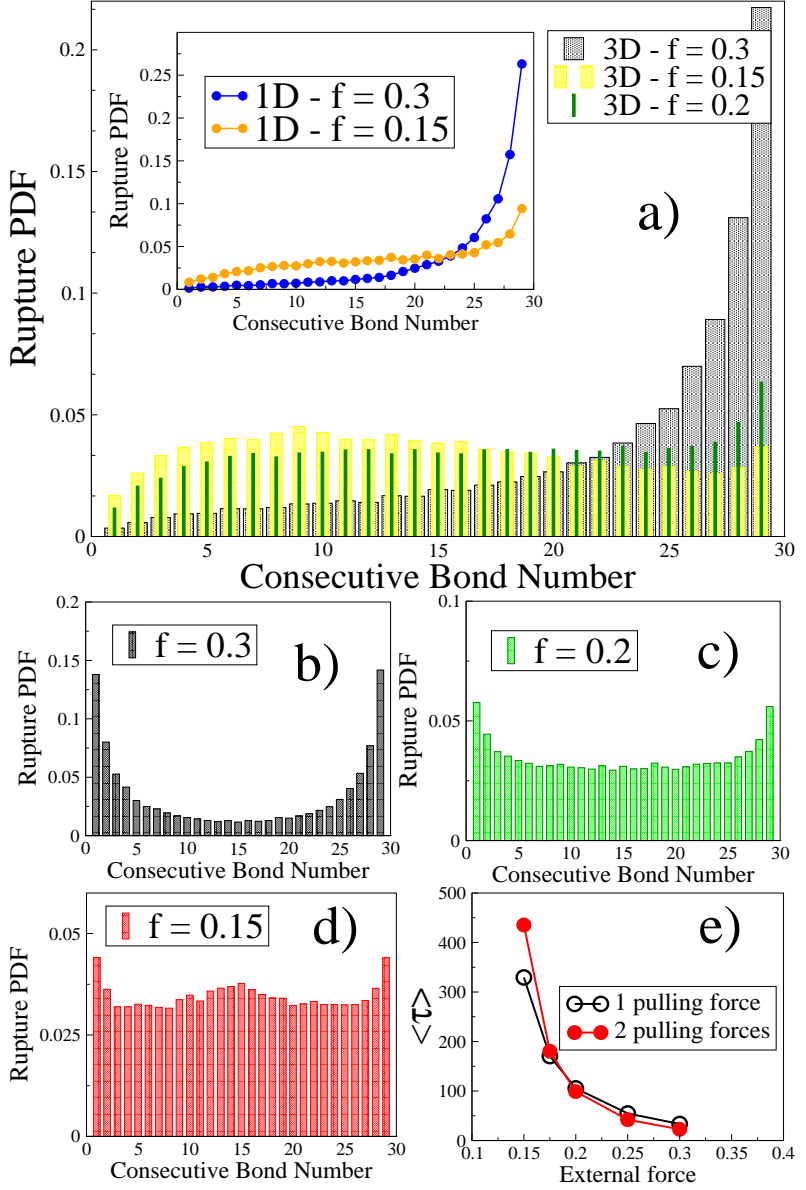

Fig. 5: a) Rupture probability histograms for $1 D$ and $3 D$ chains composed of $N=30$ for different pulling forces as indicated. b)-d) Rupture probability histograms for $3 D$ chains made of 30 particles pulled at both ends with a tensile force $f$. e) Variation of $\langle\tau\rangle$ with force $f$ for chains pulled either at the one end, or at both ends. Here $T=0.053$ and $\gamma=0.25$.

strength of the pulling force $f$, and for $f=0.15$ a local maximum in the rate builds up for the bonds that are in the middle of the chain.

How can such an inhomogeneity in the probability of bond rupture be understood? A possible explanation of the change in the location of preferential breakdown sites along the chain may be gained by Fig. 6, In this figure we present maps of the density distribution $P(x, r)$ of bead positions where $x$ is measured in direction of the pulling force $f$ whereas $r=\sqrt{y^{2}+z^{2}}$ denotes the radial component. Fig. 6] [upper panel] indicates that at high stretching $(f=0.3)$ the most probable position of the beads is along the direction of the tensile force. For $f=0.3$ (strong stretching) the chain conformation corresponds to a quasi$1 D$ structure, and the transversal fluctuations are reduced. In contrast, when the pulling force is weak $(f=0.15)$ one finds from Fig. 6 [lower panel] that the individual beads are free to make big excursions in space - $P(x, r)$ is roughly two times broader in the middle. Thus, Fig. 6 suggests that the density maps comply with the rupture 

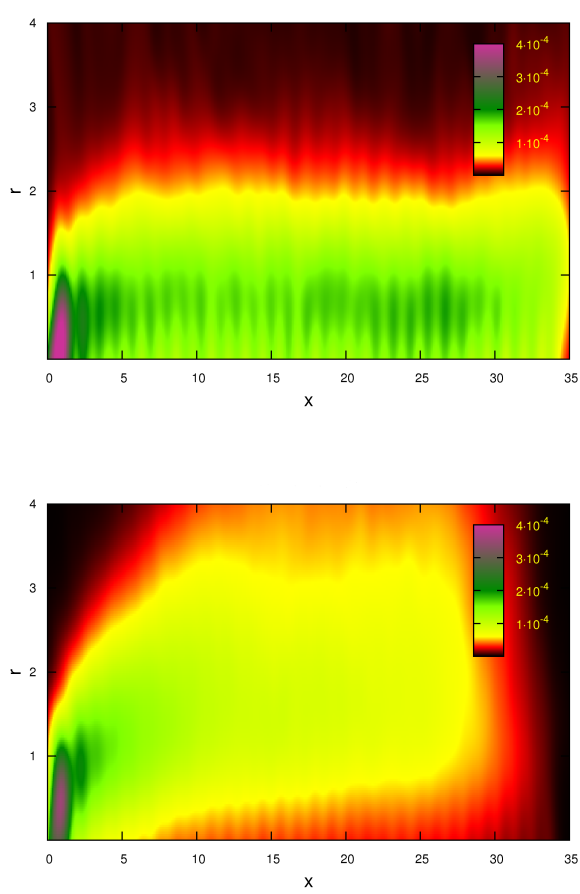

Fig. 6: Probability density distribution $P(x, r)$ of beads in a $3 D$ chain with $N=30$ particles at force: $f=0.3$ [upper panel], and $f=0.15$ [lower panel]. The $x$-axis coincides with the direction of pulling force whereas $r=\sqrt{y^{2}+z^{2}}$ denotes radial component of the bead position. Different colors indicate the value of the $\mathrm{PDF}$ as indicated in the legend. Here $T=0.053$.

histograms given in Fig. 㬏). For $f=0.3$, due to larger freedom around the pulled end, the end bonds break more easily in the terminal part of the chain. When the force is weak, $f=0.15$, the beads become more mobile around the center of the polymer which in turn leads to increased bonds scission rate there.

Chain Defects. In Fig. 7 we present the results of computer experiments concerning rupture of 30-particle chains in which a single defect is introduced. We focus on two kind of defects. First we examine the effect of an "isotope"-like defect in which a mass $m_{d}$ of the monomer located in the center of a chain is changed, while the masses of all remaining beads remain unchanged and equal to $m=1$. In Fig. 7h) we compare the rupture histogram for a chains with such a heavy/light bead to the respective scission probability distribution in a uniform system. If the central bead is replaced by a particle which is lighter/heavier than the rest of the segments, the rupture probability increases/decreases in the immediate vicinity of the introduced mass-defect. This effects a small group of beads $\sim 3$ and is pronounced only when the stretching force is sufficiently small $(f \lesssim 0.2)$. Evidently, a lighter particle can be kicked more easily by the thermostat whereby inertial effects would stretch both adjacent
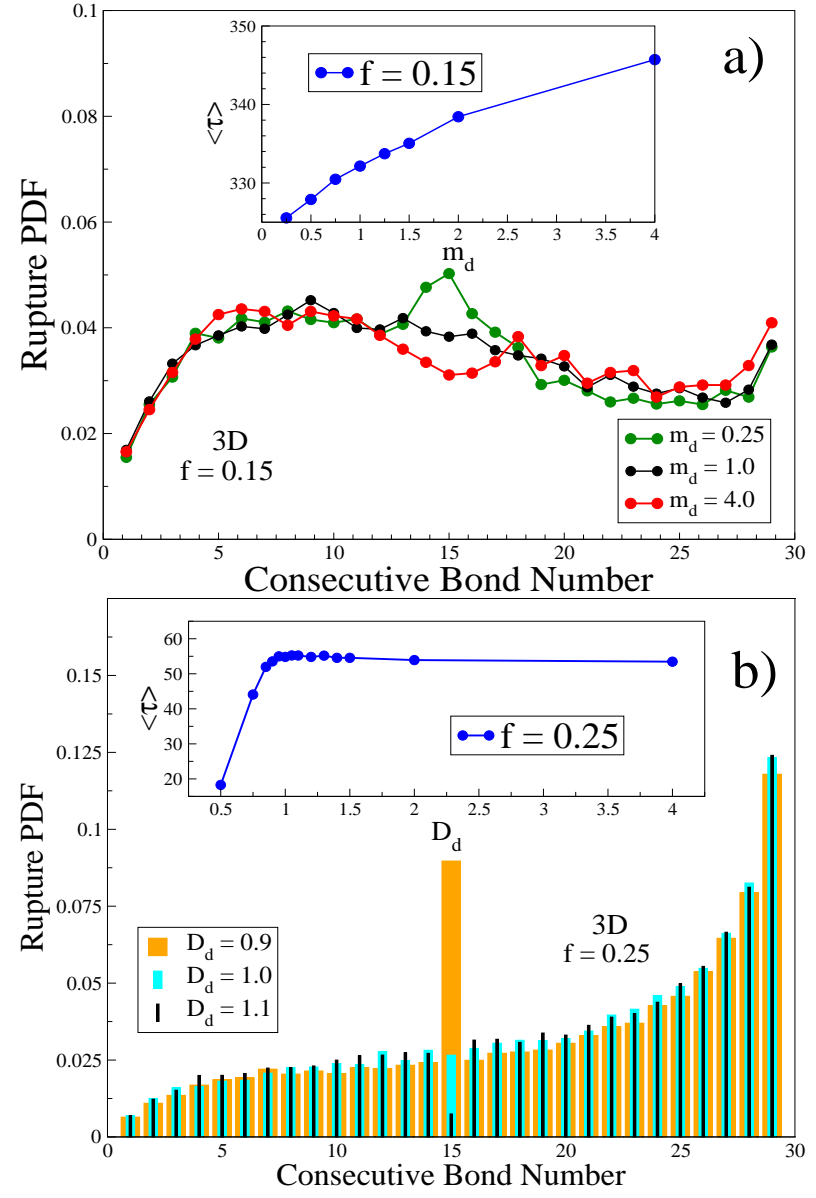

Fig. 7: a) Rupture probability histograms for 30-bead chains with a single defect bead of mass $m_{d}$ introduced in the center of a chain. Here $f=0.15$. The inset shows the mean life time time dependence on the mass of the defect. b) Rupture probability histogram for chains with a single bond defect (bond strength $D_{d}$ ) in the middle of the chain. The inset shows dependence of mean first breakage time on the strength of the defected bond. Parameters of the heat bath are: $T=0.05328$ and $\gamma=0.25$.

bonds beyond the scission threshold. In contrast, as is seen from the inset of Fig. (7 $\mathrm{k}$ ), the MFBT $\langle\tau\rangle$ grows as the defected bead becomes heavier. A defect with a larger mass is hard to accelerate, its mobility is low, and its bonds remain unstretched. In some interval of time such defect experiences many kicks which effectively cancel each other before a dangerous bond stretching occurs. Thus, the chain becomes locally more immune against breakage events which is reflected by the decrease in the probability of rupture.

As expected, the tensile strength of a particular bond (measured in units of $D$ ) affects the scission process too. To see this we varied the bond strength $D_{d}$ of the middle bond. In Fig. (7b) we present rupture histograms for chains with $N=30$ and different values of $D_{d}$. Clearly, the small variation of bond strength $( \pm 10 \%)$ results in a change of the scission probability which is located exactly at the 
defect position. In the inset of Fig. 7b) we present the MFBT $\langle\tau\rangle$ as a function of $D_{d}$. One can see that the introduction of weaker bonds $D_{d}<1$ in the chain results in a decrease of $\langle\tau\rangle$. This is due to the fact that the lifetime $\tau_{D}$ for the weak bond is very short and therefore dominates the mean $\langle\tau\rangle$. On the contrary, it appears that $\langle\tau\rangle$ is not sensitive for defects with $D_{d}>1$. In this case defected bonds appear to resist scission events which then happen predominantly in the remaining bonds.

Concluding remarks. - Our findings can be summarized as follows:

- The mean life time of the polymer chain at constant tensile force depends on chain length like $\langle\tau\rangle \propto N^{-\beta}$ whereby the power law exponent $\beta$ varies in the interval $0<\beta<1$. Generally, it appears that the exponent $\beta$ systematically declines as the external pulling force $f$ grows. This behaviour indicates a growing degree of cooperativity during the chain breakage as the pulling force $f$ is increased.

- The MFBT follows an Arhenian law $\langle\tau\rangle \propto \exp \left(E_{b} / T\right)$ whereby the effective activation barrier for scission changes with varying pulling force $f$ as $E_{b}=E_{0}-\alpha f$ in line with earlier theoretical predictions [17]. The scission times in a polymer chain under tension are exponentially distributed, $W(t) \propto \exp \left(-t / \tau\left(E_{b} / T\right)\right)$.

- The rates of bond rupture are distributed differently along the polymer backbone in the $1 D$ and $3 D$ chain models. In a $1 D$ chain the rupture rate steadily grows as one approaches the free chain end where the external pulling force is applied whereas in a $3 D$ chain bonds break predominantly in the middle of the chain. Bond rupture histograms correlate with the degree of spreading in the monomer density distribution, indicating that scissions occur most frequently in those parts of the macromolecule which undergo large fluctuations in position. Inertial effects and bead mobility provide a plausible interpretation of the observed complexity of fragmentation kinetics.

- The probability histograms for bond scission provide a clear picture of the impact of polymer defects on the fragmentation process and underline thereby the role of inertial effects. Bonds, connected to lighter segments break become a preferred site of rupture whereas heavier segment stabilize the chain.

$$
* * *
$$

J. P. would like to thank Michael Rubinstein for fruitful discussions. A. M. gratefully acknowledges support by the Max-Planck-Institute for Polymer Research during the time of this investigation. This research has been supported by the Deutsche Forschungsgemeinschaft (DFG), Grants SFB 625/B4 and FOR 597.

\section{REFERENCES}

[1] Kausch H.-H, Polymer Fracture (Springer, New York) 1987.

[2] Crist B., Annu. Rev. Mater. Sci., 25 (1995) 295.

[3] Garnier L., Gauthier-Manuel B., van der Vegte E. W., Snijders J. and Hadziloannou G., J. Chem. Phys., 113 (2000) 2497.

[4] Grandbois M., Beyer M., Rief M., ClausenSchaumann H. and Gaub H. E., Science, 283 (1999) 1727.

[5] Saitta A.M. and M. L. Klein, J. Phys. Chem. A, 105 (2001) 649.

[6] Maroja A.M., Oliveira F.A., Ciesla M. and Longa L., Phys. Rev. E, 63 (2001) 061801.

[7] Rohrig U. F. and Frank I., J. Chem. Phys., 115 (2001) 8670.

[8] Gersappe D. and Robins M. O., Europhys. Lett., 48 (1999) 150.

[9] Filippov A. E., J. Klafter, and M. Urbakh, Phys. Rev. Lett., 92 (2004) 135503.

[10] Aктah D. and Frank I., J. Am. Chem. Soc., 124 (2002) 3402.

[11] Beyer M. K. and Clausen-Schaumann H., Chem. Rev., 105 (2005) 292.

[12] Harris S. A.,Contemp. Phys., 45 (2004) 11.

[13] Oberhauser A., Hansma P., Carrion-Vazquez M. and Fernandez J., Proc. Natl. Acad. Sci. U.S.A., 98 (2001) 468.

[14] Greenleaf W., Woodside M, Abbondanzieri E. and S. Block, Phys. Rev. Lett., 95 (2005) 208102. Phys. Rev. E, 73 (2006) 050902.

[15] Suzuki Y. and Dudko O. K., Phys. Rev. Let., 104 (2010) 048101; Jour. Chem. Phys., 134 (2011) 065102.

[16] Bueche F.,J. Appl. Phys., 29 (1958) 1231.

[17] Zhurkov S.N. and Korsukov V. E., J. Polym. Sci., 12 (1974) 385.

[18] Doerr T. P. and Taylor P. L., J. Chem. Phys., 101 (1994) 10107.

[19] Lee C. F.,Phys. Rev. E, 80 (2009) 031134

[20] Stember J. N. and G. S. Ezra, Chem. Phys., 337 (2007) 11

[21] Bolton K.,Nordholm S., and Schranz H. W., J. Phys. Chem., 99 (1995) 2477.

[22] Puthur R. and Sebastian K. L., Phys. Rev. B, 66 (2002) 024304.

[23] Oliveira F. A. and Taylor P. L., J. Chem. Phys., 101 (1994) 10118.

[24] Oliveira F. A.,Phys. Rev. B, 52 (1995) 1009.

[25] Oliveira F. A. and Gonzalez J. A.,Phys. Rev. B, 54 (1996) 3954.

[26] Oliveira F. A.,Phys. Rev. B, 57 (1998) 10576.

[27] Ghosh A., Dimitrov D. I., Rostiashvili V. G., Milchev A. and Vilgis T. A., J. Chem. Phys., 132 (2010) 204902.

[28] Sain A., Dias C. L. and Grant M., Phys. Rev. E, 74 (2006) 046111.

[29] Fugmann S. and Sokolov I. M., Phys. Rev. E, 81 (2010) 031804

[30] Paturej J., Milchev A., Rostiashvili V. G. and VilGIS T. A., sumbitted to J. Chem. Phys. 